\title{
Research on Combine Harvester Positioning algorithm and Aided-navigation System
}

\author{
Jin $\mathrm{CHEN}^{1, a}$,Guangjing $\mathrm{YANG}^{2, \mathrm{~b}}, \mathrm{Kai} \mathrm{XU}^{3, \mathrm{c}}$,Yangyang CAI ${ }^{4, \mathrm{~d}}$ \\ ${ }^{1234}$ School of Mechanical Engineering, Jiangsu University, Zhenjiang, 212013, China \\ aemail:Chenjinjd@126.com, bemail:1012675546@qq.com, ${ }^{c}$ email:326511986@qq.com, \\ demail:1050256786@qq.com
}

Keywords: Combine Harvester; GPS Positioning System; Kalman Filtering; Aided-Navigation

\begin{abstract}
According to the error of the data processing algorithm applied in the GPS positioning system which is applied in agricultural machinery especially in combine harvester, the application of ARM processor is used as the data processing module and the geographical positioning information is collected by GPS module. In this paper, using dynamic Kalman Filtering algorithm based on model transformation which reduces the fluctuation range of latitude and longitude from 0.0006 and 0.0005 to 0.0003 and 0.0003 . The data can be displayed by touch screen and using positioning information and path planning algorithm can realize aided- navigation and calculation of course deviation.
\end{abstract}

\section{Introduction}

In recent years, more and more domestic and foreign universities and scientific research units have carried out some research for combine harvester positioning system, aided-navigation and automatic test production. Jiangsu University, Nantong University and Chinese Academy of Agricultural Mechanization Sciences have combined the GPS positioning system with combine harvester which realize collection of positioning information real-time and automatic navigation through the GPS positioning information[1][2][3]. In the research above-mentioned by the universities, the traditional processing method of GPS signals is Kalman filtering and Least Squares Filtering, due to the limitation of the two kinds of algorithm itself, the error of the result of positioning and navigation will be bigger. In this paper, aiming at the deficiency of the algorithm and designing a system which uses dynamic Kalman Filtering algorithm based on model transformation to process the data and the algorithm can reduce the fluctuation range of latitude and longitude data, at the same time uses ARM as the processor which can accelerate the speed of data processing. Using touch screen to display the positioning information and path information can alleviate the labor intensity of operators and the aided-navigation system can reduce the loss rate.

\section{Design of Hardware Structure}

The overall structure of the system comprises positioning module, information process module and touch screen module. The hardware diagram is shown as Figure 1: 


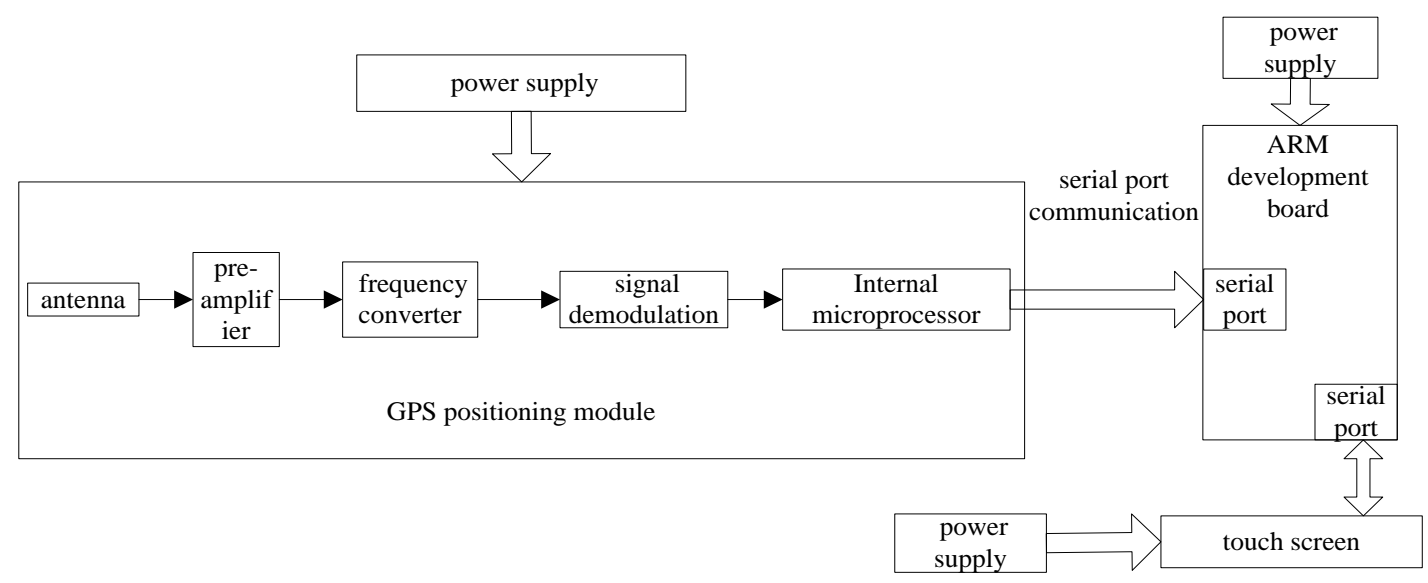

Fig.1. The Hardware Structure of System

In Figure 1, the GPS positioning system consists of three parts: the positioning information receive and transmit part; location information process part; positioning information display part. The positioning information receive and transmit part consists of GPS module and antenna with it. Information process module is composed of ARM processor and its peripheral circuit. the peripheral circuit of ARM processor is composed of power supply module, information storage unit, clock module, download module, communication module and information display module.

The hardware structure of aided-navigation system includes ARM processor, speed monitoring sensor, magnetoresistive sensor and electronic map.In the system, using magnetoresistive sensor to calculate the movement direction of the combine harvester, the principle of magnetoresistive sensor is using the principle of magnetic to measure magnetic filed component along the navigation system coordinate and get the direction of combine harvester through calculation[4].

\section{Research On Positioning Algorithm}

Traditional filtering processing method includes Kalman Filtering and Least Squares Filtering, the two methods all have disadvantages: Least Squares Filtering can only use the current measurements and can not make analysis of the current measurements. Therefore the positioning result affected by the error of measurements is relatively large and the accuracy is not high. Kalman Filtering does not need to store large amounts of data and can process the real-time dynamic data conveniently. When taking the pseudo range as observed quantity for Kalman Filtering, the initial position's approximate coordinate is need. If the approximate coordinates deviation is large, then the measurement equation will be inaccurate and result in larger discreteness. The traditional Kalman filtering algorithm for static and constant velocity motion state prediction and tracking effect is very good, but combine harvester in this paper will occur velocity change in the process of movement, its state of motion is random, so there will be deviation of model[5][6]. The change of speed occur during the operation of the combine harvester will cause the change of prediction model. When the combine harvester is working in the field, the speed is slow, so the change of speed can be approximate regard as uniform acceleration. In this paper, the dynamic Kalman Filtering algorithm based on model transformation will be used to process the data collected.[7][8].

Choosing combine harvester which in the movement and choose variable for researching:

$$
X=\left[x, v_{x}, a_{x}, \varepsilon_{x}, y, v_{y}, a_{y}, \varepsilon_{y}, z, v_{z}, a_{z}, \varepsilon_{z}\right]^{T}
$$

$$
x, v_{x}, \Delta a_{x}, \varepsilon_{x}, y, v_{y}, \Delta a_{y}, \varepsilon_{y}, z, v_{z}, \Delta a_{z}, \varepsilon_{z} \text { refer to location, speed, acceleration error and position }
$$

error.

The system's state equation can be set:

$$
\begin{aligned}
& X(t+1)=A X(t)+U(t)+W(t) \\
& U(t)=\left[\begin{array}{lllllll}
0 & 0 & \frac{\overline{a_{x}}}{\tau_{a x}} 0 & 0 & 0 & \frac{\overline{a_{y}}}{\tau_{a y}}
\end{array} 0 \begin{array}{llll}
0 & 0 & \frac{\overline{a_{z}}}{\tau_{a y}}
\end{array}\right]^{T}
\end{aligned}
$$


$A$ is system's state-transition matrix; $\tau, \tau_{\mathrm{a}}, \omega_{\mathrm{a}}, \bar{a}$ refer to time constant, acceleration time constant, white Gaussian noise and average of current acceleration.

The measurement equation can be set:

$$
Z=H X+V
$$

$H$ is observation matrix and $V$ is observation noise vector.

Setting up a adaptive Kalman Filtering equation according to (1) and (2):

$$
\begin{gathered}
\left\{\begin{array}{l}
X(k+1)=Q_{1}(k+1, k) X(k)+S(k) \\
X(k+1)=X(k+1, k)+K(k+1)[Z(k+1)-H(k+1) X(k+1, k)] \\
K(k+1)=P(k+1, k) H^{T}(k+1)\left[H(k+1) P(k+1) H^{T}(k+1)+R(k+1)\right]^{-1} \\
P(k+1, k)=\lambda(k+1) Q(k+1, k) P(k) Q^{T}(k+1, k)+F(k) \\
P(k+1)=[I-K(k+1) H(k+1)] P(k+1, k)
\end{array}\right. \\
Q_{1}(k+1, k)=\left[\begin{array}{lll}
Q_{1 x}(k+1, k) & 0 & 0 \\
0 & Q_{1 y}(k+1, k) & 0 \\
0 & 0 & Q_{1 z}(k+1, k)
\end{array}\right], Q_{1 n}=\left[\begin{array}{cccc}
0 & T & \frac{T 2}{2} & 0 \\
0 & 1 & T & 0 \\
0 & 0 & 1 & 0 \\
0 & 0 & 0 & e^{-T / \tau n}
\end{array}\right], n=(x, y, z)
\end{gathered}
$$

The average of acceleration and acceleration variance can calculated through literature, $S(k)=\left[\frac{T^{2} a_{x}}{2}, T a_{x}, \frac{T^{2} a_{y}}{2}, T a_{y}, \frac{T^{2} a_{z}}{2}, T a_{z}\right]^{T}$ is model deviation matrix of system. $Q(k+1, k) F(k) R(k) \lambda(k+1)$

refer to discrete matrix of system transfer matrix; discrete matrix of system noise covariance matrix; discrete matrix of system noise covariance matrix and adaptive forgetting factor the increase of estimation error will cause the error of variance, adaptive forgetting factor will grow, filter tracking effect is enhanced and filtering effect will be better.

\section{Design of Software}

The working process of the system is divided into three parts: data acquisition, data amplifier, data demodulation; data processing, data analysis, transferring positioning algorithm; data display and route display. The work flow chart of the whole system is shown as Figure 2: 


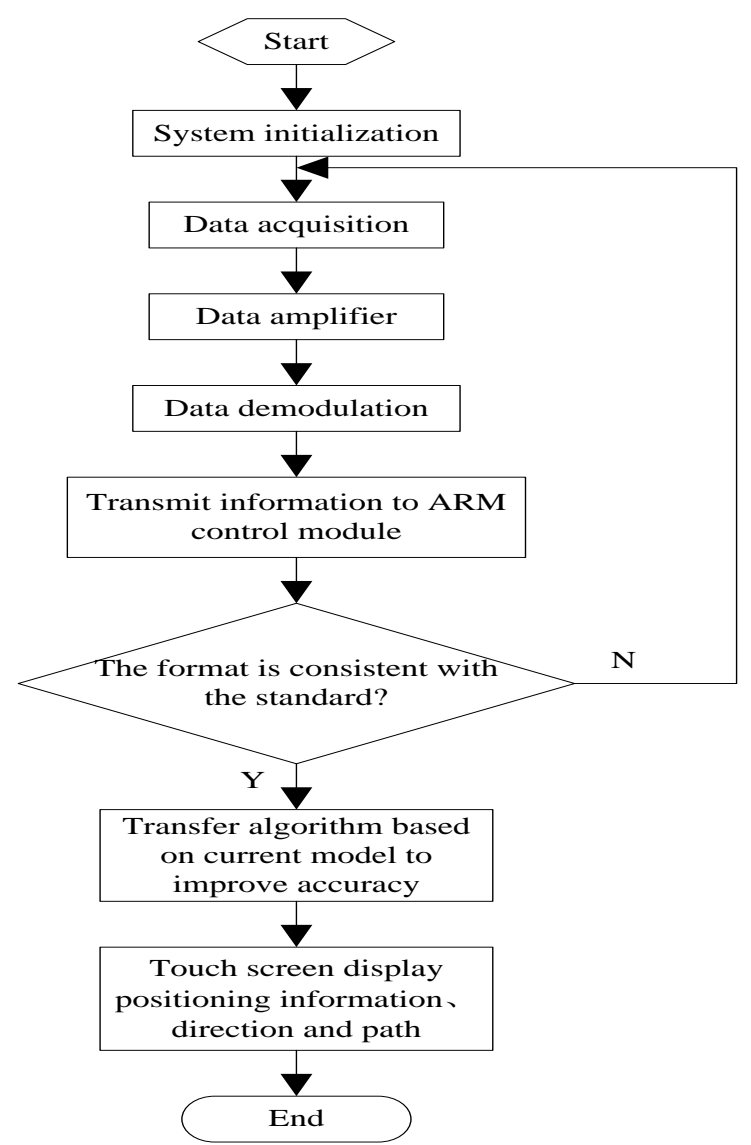

Fig.2. The software flow chart of system

When the system judge the format of the positioning information collected is accord with the standard, the system will transfer algorithm to process the information and improve the accuracy of the information. The flow chart of the algorithm is shown as figure 3:

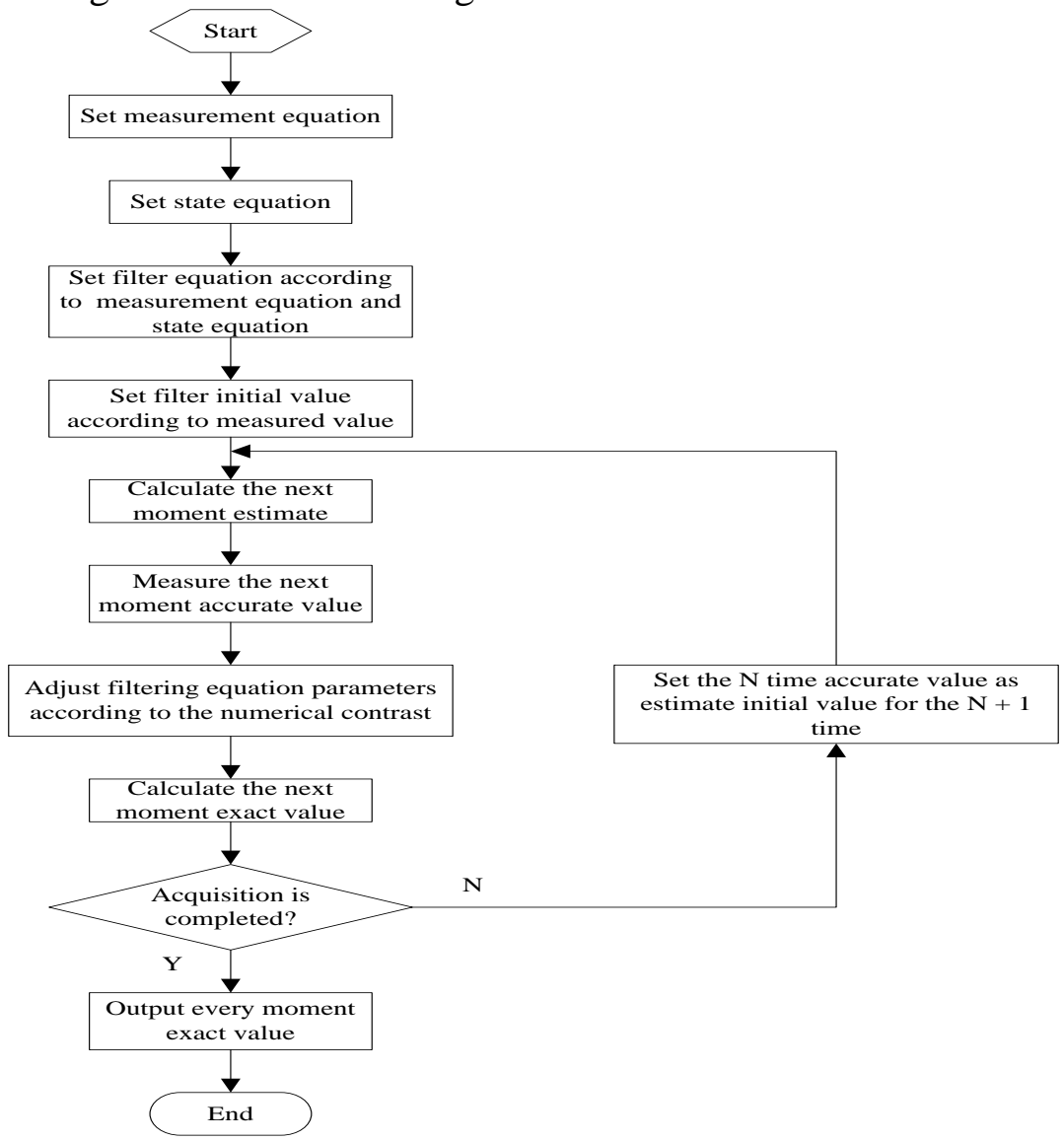

Fig.3. The flow chart of positioning algorithm 
The work of the aided-navigation system include display electronic map and calculate the shortest path. The display of electronic map is that pre-load the map into SD card before experiment, in the course of the experiment, transferring the electronic map through library function and using Qt software to realize the display of electronic map. After reading the map data, the system can realize the display of map information on touch screen through the conversion of coordinates; the calculation of the shortest path is that when the combine harvester switched to automatic control mode, the system can compare the actual routes with the set routes in the electronic map and display the direction on the touch screen. The system uses Floyd algorithm to calculate the shortest route, the algorithm is used to search for the shortest route between multi-source points in the given weighted graph. Setting four points in the experimental site's four angles and calculate the shortest route between any two points through the algorithm and electronic map[9][10].

\section{Analysis of Experimental Data}

Having experiments on the experimental site and choose the latitude and longitude data of six points in the course of speed change on either side for analysis and get a conclusion that : compared the fluctuation range of the data processed through the algorithm in this paper and the traditional Kalman Filtering algorithm, the fluctuation range reduce from 0.0006 and 0.0005 to 0.0003 and 0.0003. The comparison of longitude and latitude is shown as Figure 4 and Figure 5:

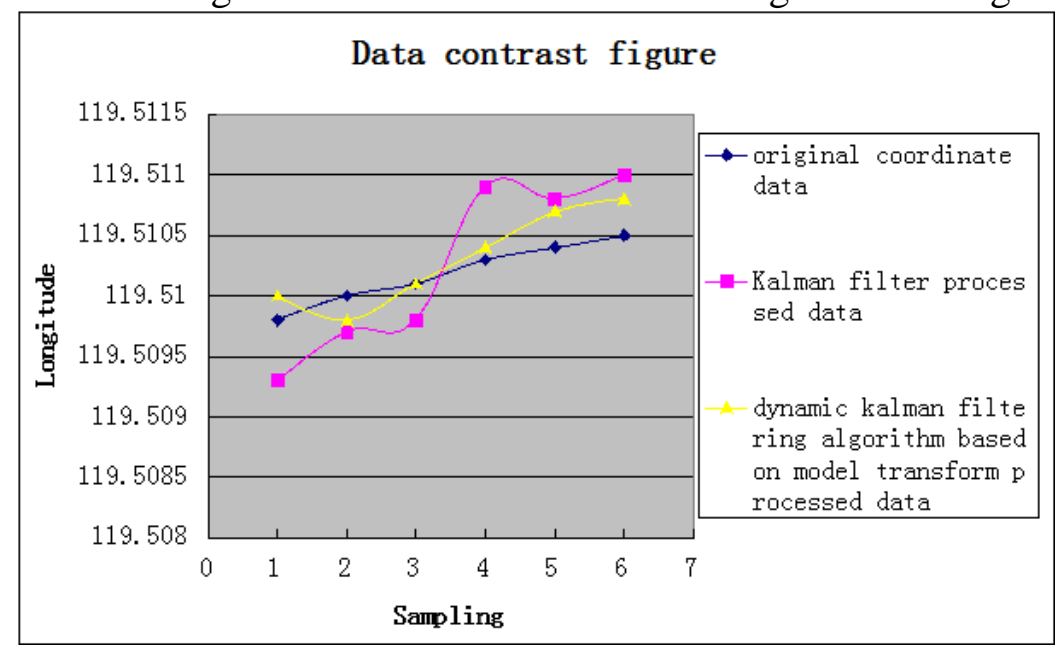

Fig.4. The comparison chart of longitude

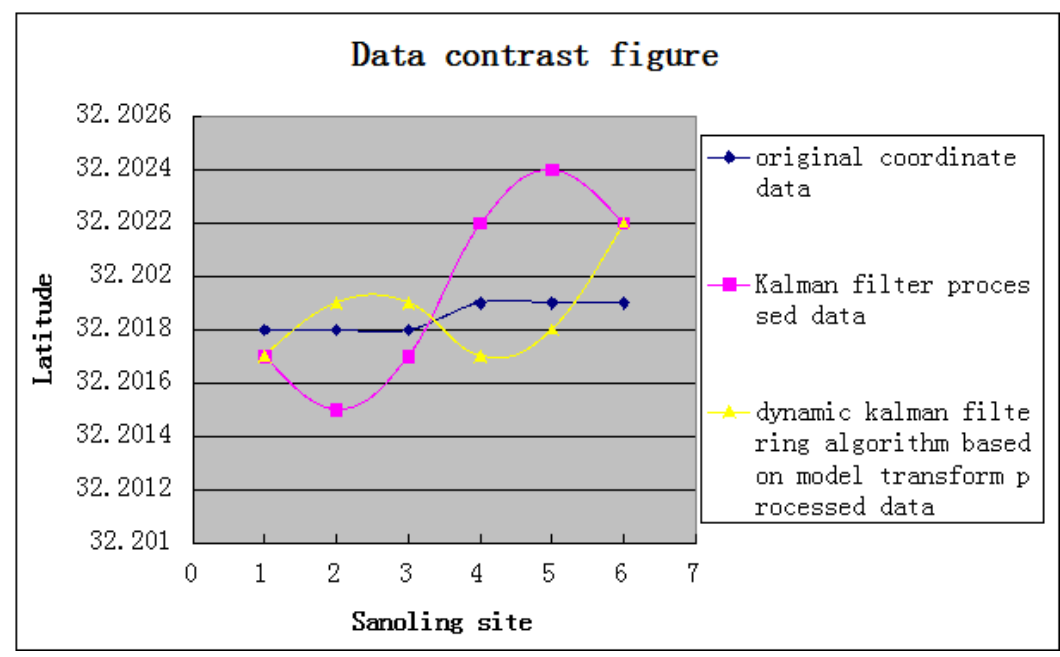

Fig.5. The comparison chart of latitude 


\section{Conclusion}

The combine harvester positioning algorithm and aided-navigation system can reduce the fluctuation range of the latitude and longitude data collected when the combine harvester is running with a non-uniform speed through reasonable design of hardware and improvement of algorithm. Designing the dynamic Kalman Filtering algorithm based on model transformation which realize the reduction of fluctuation range of the latitude and longitude data from 0.0006 and 0.0005 to 0.0003 and 0.0003 . The process of geographical location information is more accuracy when the combine harvester is running with a non-uniform speed and information can support the research of aided-navigation.

\section{Acknowledgement}

This work is supported by the National High Technology Research and Development Program of China (863 Program) (No.2012AA10A502), Science and Technology Support Project of Jiangsu province(No.BE2012312), Science and Technology Achievements Transformation Project of Jiangsu province (NO.BA2014.62), and Science and Technology Support Project of Zhenjiang city (No.NY2012028).

\section{References}

[1] Xiaoying Huang, Shuren Chen. Exploration on speed of combine harvester based on GPS technology [J]. Journal of Agricultural Mechanization Research, 2009(12).

[2] Binbin Ji, Yuping Zhang and so on. Real-time statistics of the harvesting area of combine harvester based on GPS [J]. Chinese Agricultural Mechanization, 2012(06).

[3] Yanwei Yuan. The study of GIS-based navigation and yield monitor system [D]. 2006.

[4] Zhining Hou, Zongqiang Wang, Youjun Yue. Design of vehicle GPS navigation system based on S3C2440A [J]. Journal of Tianjin University of Technology, 2010,3,(26).

[5] Chenghao Zou. Research on Kalman filtering method for the systematic errors in the GPS navigation [D]. 2012.

[6] Xu Yu, Shaoqing Yang, Sun Mu. Application of least square filter in target tracking [J]. Command Control and Simulation, 2004(04).

[7] Guo Linsong, He Yong, Zhang Qin. Real-Time Tractor Position Estimation System Using a Kalman Filter. Transactions of the CSAE. 2002.

[8] Fu Zhen, Shuman Wang, Bingjie Liu. The latest progress in adaptive Kalman filtering [J]. Tactical Missile Technology, 2009(6):62-66.

[9] Yingchun Song. Research on Kalman Filterin Kinematie Positioning

[10] Liao Xi, Kefei Yi. Development of Qt test software based on embedded Linux system [J]. Ordnance Industry Automation, 2013(08).

[11] Yuanjie Mao. Floyd algorithm for shortest path problems with MATLAB program [J]. Journal of Hebei North University, 2013. 